${ }^{1}$ Unidad de Cuidados Neurointensivos, Sanatorio Pasteur, Catamarca, Argentina. 2Unidad de Cuidados Intensivos, Hospital Hermanos Ameijeiras, La Habana, Cuba.

${ }^{3}$ Medicina Interna, Hospital de Cecina, Cecina, Italia.

${ }^{4}$ Servicio Neurología, Hospital General San Camillo de Lellis, Rieti, Italia.

${ }^{5}$ Sección Neurología, SMDN-Centro para la Prevención de Enfermedades Cardio y Cerebrovasculares, Sulmona, L'Aquila, Italia.

El presente trabajo fue efectuado sin apoyo económico alguno. Los autores declaran no tener conflicto de intereses de ninguna naturaleza.

Recibido el 27 de marzo de 2012, aceptado el 10 de julio de 2012.

Correspondencia a: Daniel Agustin Godoy Chacabuco 675. (4700) San Fernando del Valle de Catamarca. Provincia de Catamarca. Argentina. Tel: 00543834432005 Fax: 00543833432006 E-mail: dagodoytorres@yahoo. com.ar

\section{Enfoque práctico para el diagnóstico y tratamiento de los estados poliúricos en pacientes con injuria cerebral aguda}

\author{
DANIEL AGUSTÍN GODOY, ERICA ÁLVAREZ1, \\ VERÓNICA CAMPI ${ }^{1}$, CARIDAD SOLER ${ }^{2}$, \\ LUCA MASOTTI ${ }^{3}$, MARIO DI NAPOLI ${ }^{4,5}$
}

\section{Diagnosis and therapy of polyuric states in patients with acute cerebral injury}

In patients with acute cerebral injury, polyuric states can potentially trigger, maintain and aggravate the primary neurological damage, due to hypovolemia, arterial hypotension and alterations of osmolarity. The true incidence of the condition in this population is unknown. A widely validated definition of polyuric state is lacking and its etiology is multifactorial. There are two principal classes of polyuria: a) aqueous polyuria with diabetes insipidus as the main cause; and b) osmotic polyuria in which sodium, glucose or urea play the main role. Polyuric states are in close association with disorders of water and sodium metabolism and with alterations in acid-base balance. A detailed analysis of the history, clinical picture and simple laboratory determinations in blood and urine, are required for an adequate assessment of these polyuric states. The problem must be faced with pathophysiological reasoning and a systematic and sequential approach, because each disorder needs a specific therapy.

(Rev Med Chile 2013; 141: 616-625).

Key words: Diabetes insipidus; Hyponatremia; Osmolar concentration; Polyuria.
L a prevalencia de los estados poliúricos en sujetos con injuria cerebral aguda es $\checkmark$ desconocida, pudiendo presentarse como consecuencia del daño neurológico per se o por la terapéutica adoptada ${ }^{1-4}$. Poliurias pueden tener impacto negativo sobre el cerebro injuriado, a través de hipovolemia, hipotensión arterial y cambios en la osmolaridad. Implica un verdadero reto diagnóstico-terapéutico ${ }^{5,6}$; por ello, un rápido reconocimiento y adecuado análisis, permitirá instaurar la conducta más apropiada. Los estados poliúricos están relacionados con desequilibrios hidroelectrolíticos, (principalmente del contenido de sodio) y trastornos de la osmolaridad ${ }^{7-10}$, situaciones que contribuyen a agravar y perpetuar el daño inicial ${ }^{11}$. Este trabajo pretende en forma práctica y sencilla aportar un enfoque que nos permita reconocer los estados poliúricos, identificar sus causas y analizar la manera más adecuada de tratarlos.

\section{Primer paso: ¿cómo definimos poliuria?}

Poliuria deriva del griego "poly" (mucho); ouro (orina $)^{12}$. Se define de diferentes maneras: a) $>3.000 \mathrm{cc}$ diarios; b) $>150 \mathrm{ml} / \mathrm{h}$; c) $>3 \mathrm{ml} / \mathrm{kg} / \mathrm{h}$, o d) diuresis mayor a $10 \mathrm{ml} / \mathrm{min}^{5-10}$. Sus causas son variadas al igual que su duración, pudiendo estar ocasionadas por la injuria cerebral o por intervenciones terapéuticas: reposiciones de volumen, utilización de agentes osmóticos (manitol, salino hipertónico), diuréticos, nutrición parenteral. 
Conceptos fisiológicos básicos para interpretar correctamente los estados poliúricos: determinantes del volumen urinario ${ }^{7-10,13}$

El volumen y composición de la orina está determinado por:

Excreción de orina $=$ filtración glomerular + secreción tubular - reabsorción tubular.

La filtración ocurre a nivel glomerular, siendo más eficaz cuando se conservan sus propiedades intrínsecas (superficie disponible, permeabilidad) y cuando existe perfil hemodinámico adecuado. Diariamente se filtran 180 a 200 litros de plas$\mathrm{ma}^{7-10,13}$. La tasa de filtración glomerular requiere flujo sanguíneo acorde ${ }^{7-10}$. Las arteriolas glomerulares aferente y eferente poseen la propiedad de modificar su diámetro, manteniendo presión capilar y filtración constante por más que fluctúe la presión de perfusión renal. Esta capacidad, denominada autoregulación, funciona limitadamente. Por debajo del límite inferior, si desciende la presión, desciende el flujo ${ }^{7-10,13}$, ocurriendo lo contrario cuando se excede el límite superior. Sustancias de producción local o sistémica (catecolaminas, vasopresina, prostaglandinas, tromboxanos), son capaces de modificar el flujo, produciendo constricción o dilatación arteriolar ${ }^{7-10,13}$.

Los fenómenos de secreción y reabsorción ocurren a nivel tubular, resultando indispensable que cada zona conserve su permeabilidad; que las bombas de intercambio iónico funcionen correctamente y que haya respuesta adecuada a los estímulos hormonales (aldosterona, hormona anti diurética $[\mathrm{HAD}])^{7-10,13}$.

En condiciones fisiológicas, el riñón produce un adecuado balance excreción/reabsorción del sodio, lo que determina su concentración total en íntima relación con variaciones del agua corporal. Este balance depende en gran medida del estímulo simpático y de los péptidos natriuréticos ${ }^{8-10,14,15}$.

Los efectos biológicos de los péptidos natriuréticos atrial (ANP) y cerebral (BNP) incluyen vasodilatación, natriuresis y aumento del volumen urinario $^{14,15}$, mientras que el péptido $\mathrm{C}(\mathrm{CNP})$ hallado en el hipotálamo, lámina terminalis, líquido cefalorraquídeo y endotelio ${ }^{14,15}$, carece de efecto natriurético. Su rol exacto permanece por elucidar $^{14,15}$. El cerebro modula la secreción de ANP a través de vías adrenérgicas y colinérgicas, postulándose que el daño encefálico ocasiona pérdida del control secretorio, liberación excesiva de ANP y natriuresis ${ }^{14,15}$.
Del volumen plasmático filtrado en los glomérulos, $99 \%$ es reabsorbido, eliminándose $1 \%$, dando lugar a diuresis diaria de 1 a 2 litros, excretándose además una carga de solutos que oscila entre 600 a $900 \mathrm{mOsm}(1$ a $1,5 \mathrm{mOsm} / \mathrm{Kg})$ a una concentración variable entre 30 y 1.400 miliosmoles por litro de orina (mosm/l) dependiendo de la cantidad de agua libre eliminada ${ }^{7-10,13}$. En este contexto, la reabsorción tubular del agua tiene un rol clave en la composición y volumen final de la orina, dependiendo de tres factores: $:^{7-10,13}$

a) Cantidad de agua libre originada en la rama ascendente del asa de Henle, la que depende del volumen de filtrado glomerular reabsorbido a nivel proximal.

b) Permeabilidad de los túbulos distales y colectores al agua; mediante la apertura de canales específicos (acuaporinas-2) estimulados por HAD.

c) Presencia de gradiente osmótico entre la luz tubular y el intersticio medular renal (mecanismo de contracorriente a nivel asa de Henle e intercambio de urea entre estas estructuras y los túbulos colectores).

Por tanto, si carecemos de HAD; si la osmolaridad del intersticio medular renal está disminuida, o los túbulos colectores no responden al estímulo hormonal, el agua no se absorberá, dando lugar a orinas diluidas de gran volumen: poliuria acuosa ${ }^{5,6}$.

Por otra parte, cuando aumentan los solutos intratubulares que arrastran consigo agua, provocan las denominadas poliurias osmóticas ${ }^{5,6} \mathrm{como}$ sucede cuando la carga filtrada supera el umbral de reabsorción máxima tubular (ej. glucosuria de la diabetes mellitus) o las bombas que reabsorben sodio dejan de hacerlo (exceso de péptido natriurético, o están bloqueadas por diuréticos) o están funcionalmente ausentes (túbulo inmaduro durante recuperación de necrosis tubular aguda $)^{5,6}$.

\section{¿Qué consecuencias pueden acarrear al cerebro injuriado las poliurias?}

Por las razones expuestas en la Tabla 1, los individuos con lesiones cerebrales agudas, están predispuestos a desarrollar poliuria, las que pueden ocasionar hipovolemia, hipotensión arterial y trastornos hidroelectrolíticos, factores que predisponen al daño secundario ${ }^{11}$. Las agresiones sistémicas secundarias perpetúan y empeoran el daño primario, por lo tanto, resulta indispensable su prevención, detección y control ${ }^{11}$. 
Hipotensión arterial es deletérea, por ello, debemos normalizar la volemia, pilar clave que permite optimizar presión de perfusión y flujo sanguíneo cerebral ${ }^{11,16,17}$. El cerebro es muy vulnerable a disturbios del medio interno ${ }^{18,19}$. Los estados poliúricos pueden ocasionar desequilibrios en el sodio, agua, osmolaridad y distribución del contenido acuoso, originando cambios en el volumen cerebral y consecuentemente en la presión intracraneana ${ }^{18,19}$.

\section{Segundo paso: ¿cómo evaluamos los estados poliúricos?}

La pesquisa diagnóstica requiere un profundo razonamiento clínico, basado en los antecedentes, determinación del daño encefálico (examen físico, neuroimágenes) y auxilio del laboratorio ${ }^{4-6}$. La analítica debe incluir pruebas de función renal (urea, creatinina), estado ácido-base, glicemia, electrolitos (sodio, potasio, cloro, calcio, magnesio), hemograma, proteinemia y en casos seleccionados perfil hormonal (TSH, cortisol, $\mathrm{ADH}$ ). Obtener o calcular osmolaridad y analizar orina ${ }^{4-7,10,13}$. Si bien los parámetros urinarios idealmente deben obtenerse de una muestra recolectada durante $24 \mathrm{~h}$, lapsos menores $(2 \mathrm{~h})$, brindan aproximación

Tabla 1. Razones por las cuales el paciente neurocrítico es propenso a presentar alteraciones del volumen y la osmolaridad urinaria

- Alteración de conciencia
- Incapacidad para la ingesta líquida
- Daño cerebral (hipotalámico)
- Alteraciones endocrinas (ADH, péptidos natriuréticos)
- Estímulos no osmóticos de la secreción hormonal
(dolor, ansiedad)
- Reanimación con fluidos
- Tratamiento de hipertensión endocraneana (manitol,
cloruro de sodio hipertónico)
- Ventilación mecánica
- Sedoanalgesia
- Utilización de medios de contraste (TAC, angiografías)
- Diuréticos
- Interacción farmacológica

- Alteración de conciencia

- incapacidad para la ingesta liquida

- Disturbios en los mecanismos de la sed

- Alteraciones endocrinas (ADH, péptidos natriuréticos)

- Estímulos no osmóticos de la secreción hormonal (dolor, ansiedad)

- Tratamiento de hipertensión endocraneana (manitol certera de la situación, sobre todo en pacientes críticos $^{4-6}$. Es deseable que los análisis de orina y sangre sean efectuados antes de administrar fluidos o agentes con conocida influencia sobre los mismos ${ }^{4-5}$. El examen de orina debe incluir sedimento, volumen, determinación de electrolitos, $\mathrm{pH}$, urea, creatinina, glucosa, cuerpos cetónicos, densidad y osmolaridad ${ }^{4-7,10}$.

La osmolaridad urinaria puede medirse (osmómetro) o calcularse. Esta última opción es sumamente útil y práctica ${ }^{20}$.

\section{Cálculo de la Osmolaridad Urinaria $\left(\mathrm{Osm}_{u}\right)$}

Fórmula 1: Tomando como referencia densidad urinaria y del agua $(=1)$, la osmolaridad se obtiene multiplicando los últimos 2 valores de la densidad por $35^{20}$.

$$
\begin{gathered}
\operatorname{Osm}_{\mathrm{u}}(\mathrm{mOsm} / \mathrm{Kg})= \\
\left(\text { densidad orina }\left(\mathrm{d}_{\mathrm{u}}\right)-1,000\right) \times 35 .
\end{gathered}
$$

Si $\mathrm{d}_{\mathrm{u}}$ es 1.010 por ejemplo

$\mathrm{Osm}_{\mathrm{u}}=(1,010-1,000) \times 35$

$10 \times 35=350 \mathrm{mOsm} / \mathrm{kg}$.

Al utilizar esta fórmula, tener en cuenta correcciones en $d_{u}$ si detectamos glicosuria $y / o$ proteinuria.

Por cada g/dl de glucosuria, disminuir $\mathrm{d}_{u} 0,004$

Por cada $\mathrm{g} / \mathrm{dl}$ de proteinuria, descender $\mathrm{d}_{\mathrm{u}}$ 0,003

Esta fórmula se INVALIDA cuando se administra manitol, piperacilina, carbenicilina, o carbapenems ${ }^{20}$.

Fórmula 2: Obtención de osmolaridad a través de la determinación de electrolitos y urea urinarios, (descartar glucosuria $)^{20}$.

$$
\begin{gathered}
\operatorname{Osm}_{\mathrm{u}}(\mathrm{mOsm} / \mathrm{kg})= \\
\left(\mathrm{Na}_{\mathrm{u}}^{+}+\mathrm{K}_{\mathrm{u}}^{+}\right) \times 2+\left(\text { Urea }_{\mathrm{u}} / 5,6\right)
\end{gathered}
$$

Otros conceptos a destacar son:

Clearance osmolar: La cuantificación de la excreción de agua, se realiza dividiendo el volumen urinario $\left(\mathrm{Vol}_{\mathrm{u}}\right)$ en dos componentes:

a) $\mathrm{Vol}_{u}$ necesario para excretar solutos a la concentración plasmática (isotónico); se denomina Clearance osmolar (Cosm $)^{4-7,10}$.

$$
\frac{\operatorname{Cosm}=\operatorname{Osm}_{\mathrm{u}} \times \mathrm{Vol}_{\mathrm{u}}}{\text { Osmolaridad plasmática }\left(\mathrm{Osm}_{\mathrm{pl}}\right)}
$$


b) Clearance de agua libre $\left(\mathrm{CH}_{2} \mathrm{O}\right)$, definido como el volumen de agua libre de solutos que se suma o resta a la porción isotónica de la orina (Cosm) para generar orina hipotónica o hipertónica ${ }^{4-7}$.

$\mathrm{CH}_{2} \mathrm{O}=\mathrm{Vol}_{\mathrm{u}}-\mathrm{Cosm}$ (orina hipotónica)

$\mathrm{CH}_{2}^{2} \mathrm{O}=\mathrm{Cosm}-\mathrm{Vol}_{\mathrm{u}}$ (orina hipertónica)

$\mathrm{Osm} \mathrm{u}=\mathrm{Osm} \mathrm{p} \mathrm{y} \mathrm{CH}_{2} \mathrm{O}$ no existe (orina isotónica)

Fracción excretada de agua libre $\left(\mathrm{FEH}_{2} \mathrm{O}\right)$ : cantidad de agua excretada en la orina en relación a la filtrada en el glomérulo. Se calcula mediante la relación creatinina plasmática $(\mathrm{Cr} \mathrm{pl})$ y urinaria $\left(\mathrm{Cr}_{\mathrm{u}}\right)^{4-7,10,13}$.

$\mathrm{FEH}_{2} \mathrm{O}=\mathrm{Cr} \mathrm{pl} / \mathrm{Cr}_{\mathrm{u}}$

Fracción osmolar excretada (FEosm): cuantifica los solutos eliminados en relación a los filtrados en el glomérulo. Se calcula mediante la relación Cosm/clearance de creatinina ${ }^{7,8}$.

\section{Tercer paso: clasificación de los estados poliúricos}

Existen diversas formas de clasificar las poliurias $^{4-6,13,21}$. Poliuria adecuada es aquella secundaria a excesivo ingreso de agua y/o solutos (resucitación politraumatizado $)^{4-6}$. Inadecuada es cuando sobreviene como consecuencia de desequilibrios en el balance de agua y solutos (diuréticos) ${ }^{4-6,13,21}$.

En primera instancia, debemos diferenciar si la poliuria es provocada por excesiva eliminación de solutos o agua, para ello, lo más práctico es clasificarlas de acuerdo a la osm $\mathrm{yd}_{\mathrm{u}}^{4-6,8}$ (Figura 1).
Poliurias acuosas son aquellas en donde la fuerza impulsora es el agua, caracterizándose $\mathrm{Osm}_{\mathrm{u}}$ $<150 \mathrm{mOsm} / \mathrm{kg} \mathrm{y} \mathrm{d}_{\mathrm{u}}<1.010^{4-6,8}$.

Poliurias osmóticas son aquellas ocasionadas por excreción de solutos en cantidades mayores a lo normal, es decir, > $1.000 \mathrm{mOsmol} /$ día. Sus rasgos distintivos son $\mathrm{Osm}_{\mathrm{u}}>300 \mathrm{mOsm} / \mathrm{kg}$ y $\mathrm{d}_{\mathrm{u}}>1.015^{4-6,8,9}$. Los solutos pueden agruparse en: electrolitos (sodio, calcio, bicarbonato) o no electrolitos (glucosa, urea, manitol) ${ }^{6}$.

Finalmente, un tercer grupo de poliurias son las denominadas mixtas, compartiendo características de poliurias acuosas y osmóticas $\left(\mathrm{Osm}_{\mathrm{u}}\right.$ 150-300 mosm $\left./ \mathrm{kg} ; \delta_{\mathrm{u}} 1.010-1.015\right)^{6}$.

Los criterios que definen los tipos de poliuria se resumen en la Tabla 2.

\section{Cuarto paso: análisis fisiopatológico}

\section{a. Poliurias acuosas $3-5,22-24$}

Dos situaciones son posibles (Figura 2):

- Poliuria psicógena, trastorno psiquiátrico inducida por excesiva ingesta de agua ${ }^{4,5}$.

- Poliuria verdadera o diabetes insípida (DI): de mayor prevalencia durante injuria cerebral; $3,7 \%$ en unidades neuroquirúrgicas; $26 \%$ en trauma craneoencefálico grave $e^{1,3,5,22-26}$. Puede tener origen central resultado del déficit completo o parcial de HAD, o nefrogénico, en donde la concentración de HAD es normal, pero los túbulos distales y colectores no responden a dicha hormona $a^{3,5,22-26}$. Las etiologías se enumeran en la Tabla 3.

En la DI central, la alteración puede encontrarse a cualquier nivel, tanto en la producción como en la liberación de HAD, mientras que la

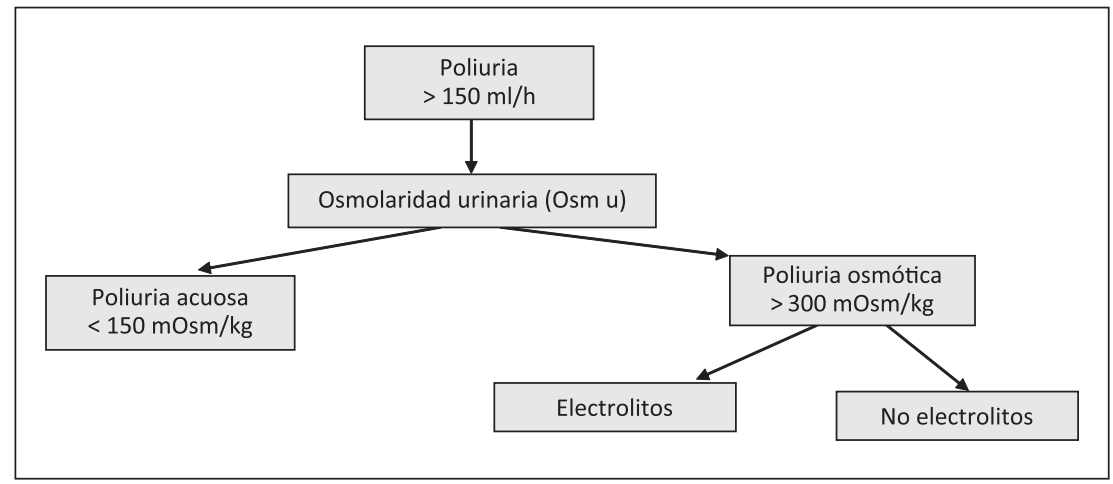

Figura 1. Clasificación de los estados poliúricos; $\mathrm{mOsm} / \mathrm{kg}$ : miliosmoles por kilogramo. Osmolaridad urinaria $=\left[\mathrm{Na}^{+}\right.$ $\left.\left.+\mathrm{K}^{+}\right) \times 2\right]+$ Urea $_{\mathrm{u}} / 5,6$. 
Poliurias: diagnóstico y tratamiento - D. A. Godoy et al

Tabla 2. Cuadro comparativo de los criterios diagnósticos en los distintos tipos de poliurias

\begin{tabular}{|lccc|}
\hline & Poliuria acuosa & Poliuria osmótica & Poliuria mixta \\
\hline Osmolaridad uinaria mOsm/kg & $<150$ & $>300$ & 150 a 300 \\
\hline Densidad urinaria & $<1.010$ & $>1.015$ & 1.010 a 1.015 \\
Relación osmolaridad urinaria/osmolaridad plasmática & $<1$ & $>1$ & $<1$ \\
Fracción excretada osmolar "FEosm"(C osmolar / Cl creatinina) & $<3,5$ & $>3,5$ & $>3,5$ \\
Clearance de agua libre "CH20" (volumen urinario - Cosm) & $>0$ & $<0$ & $>0$ \\
\hline
\end{tabular}

mOsm/kg: miliosmoles por kilogramo; C osmolar: clearence osmolar; Cl creatinina: clearence creatinina; Osmolaridad plasmática total $=\left(\mathrm{Na}^{+}+\mathrm{K}^{+}\right) \times 2+$ Glicemia/18 + BUN/2,8. Los electrolitos se multiplican por el factor 2 porque se considera con ello los aniones asociados, fundamentalmente cloro y bicarbonato. En situación fisiológica con valores normales de urea y glucosa, (las cuales se distribuyen y equilibran rápidamente entre los compartimentos del medio interno), la osmolaridad como depende del número de partículas osmóticamente activas, depende de los electrolitos antes mencionados (osmolaridad electrolítica). En cambio cuando los valores de glicemia y uremia están incrementados comienzan a jugar un papel preponderante (osmolaridad no electrolítica). Glicemia se divide por el factor 18 porque $1 \mathrm{mosm}$ glucosa $=18 \mathrm{mg} / \mathrm{dl}$. Nitrógeno ureico (BUN) se divide por 2.8 porque 1 mosm nitrógeno ureico $=2,8 \mathrm{mg} / \mathrm{dl}$.

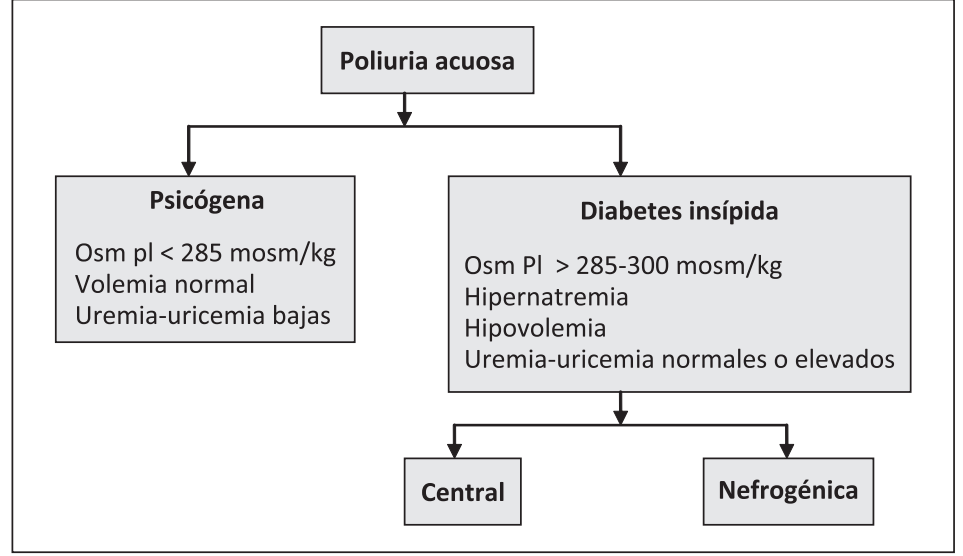

Figura 2. Diagnóstico diferencial de las Poliurias Acuosas. Osm pl: osmolaridad plasmática; mosm/kg: miliosmoles por kilogramo.

\section{Tabla 3. Diabetes insípida: tipos y etiologías}

\section{Central o neurogénica (deficiencia de HAD)}

- Traumatismo encefalocraneano

- Tumores (craneofaringiomas, meningiomas, adenomas de hipófisis, linfomas, metástasis)

- Granulomas (sarcoidosis, histiocitosis)

- Infecciones del SNC (meningitis, encefalitis)

- Apoplejia pituitaria (Síndrome de Sheehan)

- Hemorragia subaracnoidea

- Enfermedades autoinmunes (neurolupus)

- Posoperatorio cirugía de revascularización miocárdica

\section{Nefrogénica (resistencia a la HAD)}

- Metabólicas (hipokalemia, hipercalciuria)

- Tóxicas (litio, metoxifluorano)

- Anemia de células falciformes

- Sarcoidosis

- Enfermedad renal poliquística

- Pielonefritis

- Post-obstructiva

SNC: sistema nervioso central; HAD: hormona antidiurética.
DI nefrogénica se encuentra asociada a situaciones que alteran la generación y mantenimiento del gradiente osmótico medular renal ${ }^{3,5,22-26}$.

En la DI central, la diuresis diaria excede 6-7 litros, no responde a restricción de volumen ${ }^{7,9}$, osm $_{u}<150 \mathrm{mOsm} / \mathrm{kg} ; \mathrm{d}_{\mathrm{u}}<1.005$, acompañadas de hipovolemia, natremias $>155 \mathrm{mEq} / \mathrm{L}_{\text {y osm }}$ $>305 \mathrm{mmol} / \mathrm{kg}^{3,5,22-26}$. Hipernatremia factor de riesgo independiente de mortalidad, empeora el pronóstico ${ }^{17,27-31}$.

El diagnóstico diferencial entre las DI se fundamenta en los antecedentes, etiologías desencadenantes, concentración de HAD y pruebas terapéuticas ${ }^{3-5,22-25}$.

En la DI central la concentración de HAD está muy descendida o indetectable (normal 0,7-3,8 picogramos $/ \mathrm{ml}$ ), mientras que en la nefrogénica son normales ${ }^{3-5,22-25}$. Otra forma de diferenciarlas 
es mediante los test de deprivación de agua y administración de vasopresina o desmopresina ${ }^{3-5,22-25}$.

\section{Test de restricción hídrica ${ }^{3-5,22-25}$}

El test de deprivación de agua está basado en lograr deshidratación, máximo estímulo para la liberación de HAD. Usualmente limitado por la sed, hipotensión arterial u otra manifestación clínica de deshidratación por lo que su utilidad es limitada durante injuria encefálica. Requiere estricta vigilancia y medición horaria del peso, osmolaridad y densidad urinaria.

Al obtener 2 osm $_{u}$ consecutivas con variaciones $<30 \mathrm{mOsm} / \mathrm{kg}$ o el peso corporal desciende más de $3 \%$, administrar subcutáneamente 5 U HAD. Luego de $1 \mathrm{~h}$ medir osm $\mathrm{u}^{\text {. }}$

La respuesta normal incrementa $\operatorname{osm}_{\mathrm{u}}$ 2-4 más que la plasmática. Administrar HAD provoca aumento adicional $<$ al 9\%.

En DI central completa, el test evidencia que la osm $_{\mathrm{u}}$ no es $>\mathrm{osm}_{\mathrm{pl}}(\leq 300 \mathrm{mosm} / \mathrm{l})$. Suministrando HAD incrementa osm $>50 \%$.

En DI nefrogénica, la respuesta al test muestra $\mathrm{osm}_{\mathrm{pl}}>295$ mosm/l con osm $\mathrm{u}$ oscilando entre 300-700 mosm/l. ADH exógena no modifican los valores antedichos.

\section{b. Poliurias osmóticas}

Se clasifican de acuerdo al mecanismo de producción en ${ }^{6,8}$ (Figura 3):

1. Tubulares.

2. Hiperflujo de solutos.

3. Hemodinámicas.

\section{Tubulares}

Caracterizadas por incapacidad de reabsorción tubular de agua y solutos ${ }^{6,8}$. Entre sus causas, po- demos citar: diuréticos, estados pseudodiuréticos, disfunción renal en etapa poliúrica, y expansión del espacio extracelular (EEC) $)^{2,6,8}$, favorecida por inflamación, daño endotelial e incremento de la permeabilidad capilar ${ }^{32,33}$. En la fase de recuperación se elimina el volumen retenido, ocasionando pérdida de sodio por inhibirse su reabsorción: "poliurias de recuperación". Los estados "pseudodiuréticos" se asocian a hipercalcemia, uso de drogas o proteínas catiónicas (aminoglucósidos, mieloma) y excreción de aniones no reabsorbibles diferentes al cloro (bicarbonato) ${ }^{2,6}$.

En los pacientes con neuroinjuria, el síndrome de pérdida renal de sal de origen cerebral (SPRSOC) es una causa reconocida de pérdida de sodio ${ }^{2,15,18,19,34-36}$ que no debe confundirse con el síndrome de secreción inapropiada de $\operatorname{HAD}^{2,18,19,34-36}$ (Tabla 4).

Tabla 4. Diagnóstico diferencial de los principales estados asociados con disnatremias durante neuroinjuria grave

\begin{tabular}{|lccc|}
\hline & SPRSOC & SIADH & DIC \\
Balance hídrico & $\downarrow$ & $\rightarrow$ ó $\uparrow$ & $\rightarrow$ ó $\downarrow$ \\
Diuresis & $\uparrow \uparrow$ & $\rightarrow$ ó $\downarrow$ & $\uparrow \uparrow \uparrow$ \\
Natremia & $\downarrow$ ó & $\downarrow$ & \\
Natriuresis & $\uparrow$ & $\uparrow$ & $\rightarrow$ ó $\downarrow$ \\
Osmolaridad plasmática & $\downarrow$ & $\downarrow$ & $\uparrow$ \\
ADH & $\uparrow o ́ ~$ & $\uparrow$ & $\downarrow \downarrow$ \\
\hline
\end{tabular}

Nótese que en el SIHAD, la poliuria no es un pilar diagnóstico, por lo que su existencia prácticamente lo descarta. ADH: hormona antidiurética. SPRSOC: síndrome de pérdida renal de sal de origen cerebral; DIC: diabetes insípida central; SIHAD: secreción inadecuada de ADH.

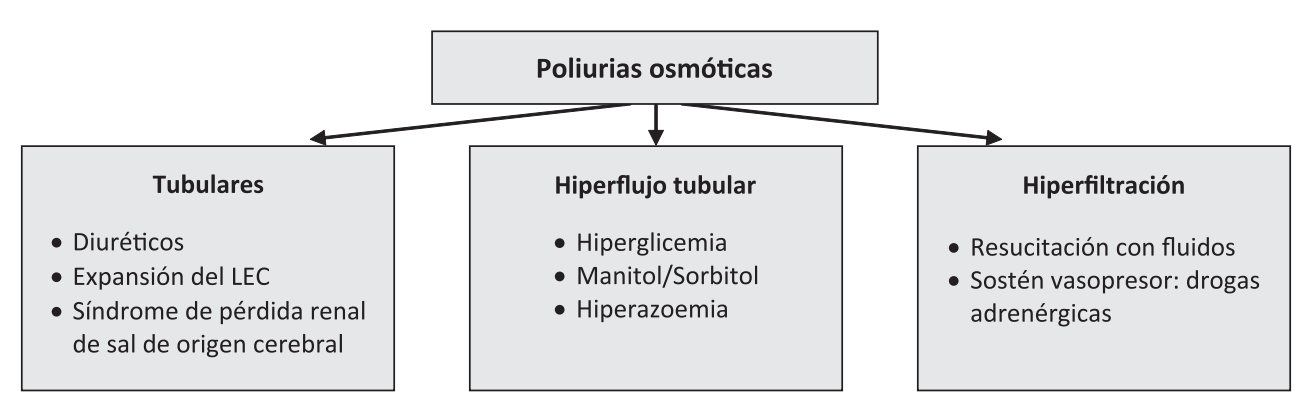

Figura 3. Etiologías más frecuentes de las poliurias osmóticas según su mecanismo de producción. LEC: líquido extracelular. 


\section{Hiperflujo tubular de solutos}

Se caracterizan por excreción de moléculas osmóticas que arrastran agua, dando lugar a orinas hipertónicas ${ }^{6,8,10}$. Los solutos más frecuentes son:

Glucosa: glicemias $>1,80-2,0 \mathrm{~g} / \mathrm{L}$, generan poliuria por superar la absorción máxima en los túbulos contorneados proximales. Causas: diabetes mellitus, respuesta a la injuria, aporte excesivo (hiperalimentación parenteral) $^{6}$.

Urea: diariamente se eliminan 12 a 20 g. Es necesario para desencadenar poliuria: a) hiperazoemia, hipercatabolismo e injuria renal aguda (necrosis tubular, rabdomiolisis, sepsis); o b) obstrucción de vías urinarias (cálculos, tumores), las que al aliviarse produce incremento del flujo tubular de urea ${ }^{6}$.

Otras: soluciones hipertónicas (manitol, cloruro de sodio); diuréticos ${ }^{37-40}$.

\section{Hemodinámicas}

Los mecanismos generadores son hiperflujo tubular e hiperfiltración glomerular ${ }^{2,8,10}$. En general no modifican $\mathrm{osm}_{\mathrm{pl}}$, natremia ni equilibrio ácido-base. Tampoco ocasionan hipovolemia ${ }^{2,8,10}$. Durante resucitación, el aporte de fluidos y vasopresores (noradrenalina), incrementan filtración glomerular, principalmente del sodio. Es una forma de poliuria apropiada ${ }^{2,8,10}$.

Las catecolaminas generan natriuresis por distintos mecanismos: a) incremento del tono venoso, presión de llenado ventricular y liberación de péptido natriurético ${ }^{14,15}$; b) efecto inotrópico directo; c) vasodilatación renal con aumento del flujo sanguíneo e inhibición de la reabsorción tubular de sodio y de la secreción de aldosterona (dopamina) $)^{2,10}$.

Otra forma de clasificarlas es de acuerdo al soluto excretado: electrolitos, o no electrolitos (abordadas anteriormente $^{6,8,10} \mathrm{Al}$ analizar las poliurias por electrolitos, debemos conocer su excreción diaria, $\mathrm{pH}$ y anión restante urinario $(\mathrm{AGU})^{6}$ (Tabla 5). Consideraremos las de mayor prevalencia y relevancia.

Cloruro de sodio: La más frecuente. La fuerza impulsora la otorga el poder de arrastre de agua que la sal tiene. Etiológicamente destacan: a) infusión de cloruro de sodio iso o hipertónico ${ }^{6,8,10,17,37}$; b) diuréticos (furosemida) ${ }^{6,8} ; \mathrm{c}$ ) síndrome de pérdida renal de sal de origen cerebral ${ }^{2,34}$; d) insuficiencia suprarrenal ${ }^{26} ;$ d) nefropatías perdedoras de sal ${ }^{6,8}$; e) fase de recuperación de injuria renal aguda $a^{6,8}$.
Bicarbonato de sodio: Para diagnosticar este tipo de poliurias resulta indispensable obtener y analizar estado ácido base, anión restante (AGU) y $\mathrm{pH}$ urinario ${ }^{6}$. Niveles de AGU $>70 \mathrm{mEq} / \mathrm{L}$ pueden estar ocasionados por cloro o bicarbonato ${ }^{6}$. Obtener $\mathrm{pH}_{\mathrm{U}}>7,5$ indica pérdida de bicarbonato ${ }^{6}$. Posibles causas: a) administración de bicarbonato; b) acidosis tubular renal; c) síndrome de lisis tumoral; d) acetazolamida.

Calcio: Puede tener fuerzas impulsoras diferentes que se potencian entre sí. Hipercalciuria ocasiona poliuria acuosa (diabetes insípida nefrogénica) o por solutos, en cuyo caso el acompañante obligado es el sodio ${ }^{6}$.

$\mathrm{Su}$ incidencia es baja, Tener en mente en pacientes oncológicos, por el uso prolongado de corticoides, furosemida o quimioterapia (síndrome lisis tumoral).

\section{Quinto paso: establecer relación entre poliurias y disnatremias}

El sodio plasmático, es clave en el enfoque diagnóstico de las poliurias ${ }^{5-9}$. Cuando la excreción de agua libre de solutos no es acompañada de aporte adecuado, inevitablemente se desarrollará hipernatremia ${ }^{6-9,22-29}$, mientras que cuando no es posible eliminar el agua libre o hay un exceso de aporte, sobrevendrá hiponatremia ${ }^{5-9}$.

La asociación poliuria-hiponatremia está presente en las poliurias tubulares por incapacidad

\section{Tabla 5. Excreción urinaria diaria de electrolitos ${ }^{6,41}$}

\begin{tabular}{|c|c|}
\hline - $\mathrm{Na}^{+}$ & : 40-250 mEq/L \\
\hline$\cdot \mathrm{Cl}^{-}$ & : 110-250 mEq/L \\
\hline \multicolumn{2}{|c|}{ - $\mathrm{HCO}_{3}{ }^{-}: 0-0,1 \mathrm{mEq} / \mathrm{L}$} \\
\hline \multicolumn{2}{|c|}{ - $\mathrm{Ca}^{++}:<4 \mathrm{mg} / \mathrm{kg}$} \\
\hline \multicolumn{2}{|c|}{ - $\mathrm{pH} \quad: 4,6-7,5$} \\
\hline \multicolumn{2}{|c|}{$\begin{array}{l}\text { - Anión-Gap urinario: }\left(\mathrm{Na}_{u}^{+}+\mathrm{K}_{u}^{+}\right)-\mathrm{Cl}_{u}^{-} \\
\text {Entre -50 y } 70 \mathrm{mEq} / \mathrm{L} \text { Diuresis por Cloruro de sodio } \\
<-50 \text { Diuresis por cationes diferentes al sodio o } \\
\text { potasio por ejemplo } \mathrm{Ca}^{++}, \mathrm{NH}_{4} \\
>70 \text { Diuresis por aniones no cloro, por ejemplo bicar- } \\
\text { bonato, salicilatos, cetoácidos }\end{array}$} \\
\hline $\begin{aligned} \text { - Electr } \\
\\
2 \times(\mathrm{N}\end{aligned}$ & $\begin{array}{l}\text { olitos urinarios totales }\left(\mathrm{E}_{\mathrm{u}}\right) \text { : } \\
\left.\mathrm{a}^{+}{ }_{\mathrm{u}}+\mathrm{K}^{+}{ }_{\mathrm{u}}\right) \times \text { Volumen urinario }\end{array}$ \\
\hline
\end{tabular}


en la reabsorción del sodio (síndrome de pérdida renal de sal de origen cerebral, diuréticos, estados pseudodiuréticos) $)^{1,2,4,18,19,34}$.

Habitualmente, las poliurias acuosas están vinculadas a estados hipertónicos 1,3,5,8,22-25 $^{\text {. }}$

Las poliurias por hiperflujo tubular pueden coexistir con cualquier valor de natremia ${ }^{6,42,43}$.

A continuación, delineamos consideraciones prácticas para el manejo de las poliurias posreanimación:

- Si la natremia asciende o es $>160 \mathrm{mEq} / \mathrm{L}$, conjuntamente con poliuria acuosa, debemos pensar en la posibilidad de DI central o nefrogénica ${ }^{1,3,5,22-26,29}$. No restringir fluidos, reemplazar las pérdidas. Evitar hipotensión e hipovolemia. Administrar vasopresina o desmopresina con lo cual haremos el diagnóstico y la diferenciación entre ambos tipos de $\mathrm{DI}^{1,3,5,22-26,29}$ (Tabla 6).

- Si la natremia es normal, puede tratarse de poliuria por expansión del EEC o por presión. En estos casos deberá evitarse la generación renal de agua libre de electrolitos, por ello, algunos autores aconsejan utilizar solución salina al 3\%, para evitar hiponatremia posreanimación ${ }^{26}$.

\section{Tabla 6. Manejo terapéutico de la diabetes insipida de origen central}

- Reemplazar las pérdidas, evitar hipovolemia e hipotensión

- Calcular déficit de Agua mediante la siguiente fórmula: Agua a reponer (litros) $=\left(\mathrm{Na}^{+}\right.$inicial $\times \mathrm{ACT} / \mathrm{Na}^{+}$ deseado) - ACT

$\mathrm{ACT}=$ Agua corporal total $=0,6 \times \mathrm{kg}$ de peso

- Evitar hiperglucemia y sobrecarga de volumen

- Corregir lentamente hipernatremia, recomendable a una tasa no > a 0,5 mEq por $\mathrm{h}$

- Acetato de desmopresina intravenoso o subcutáneo (4 $\mathrm{mcg} / \mathrm{ml}$ ) a razón de 0,5 - $1 \mathrm{ml}, 2$ ó 3 veces en el día. La dosis debe ser individualizada de acuerdo a la respuesta clínica y de laboratorio.

- Monitorizar $\mathrm{Na}^{+}, \mathrm{K}^{+}$, diuresis horaria y densidad urinaria

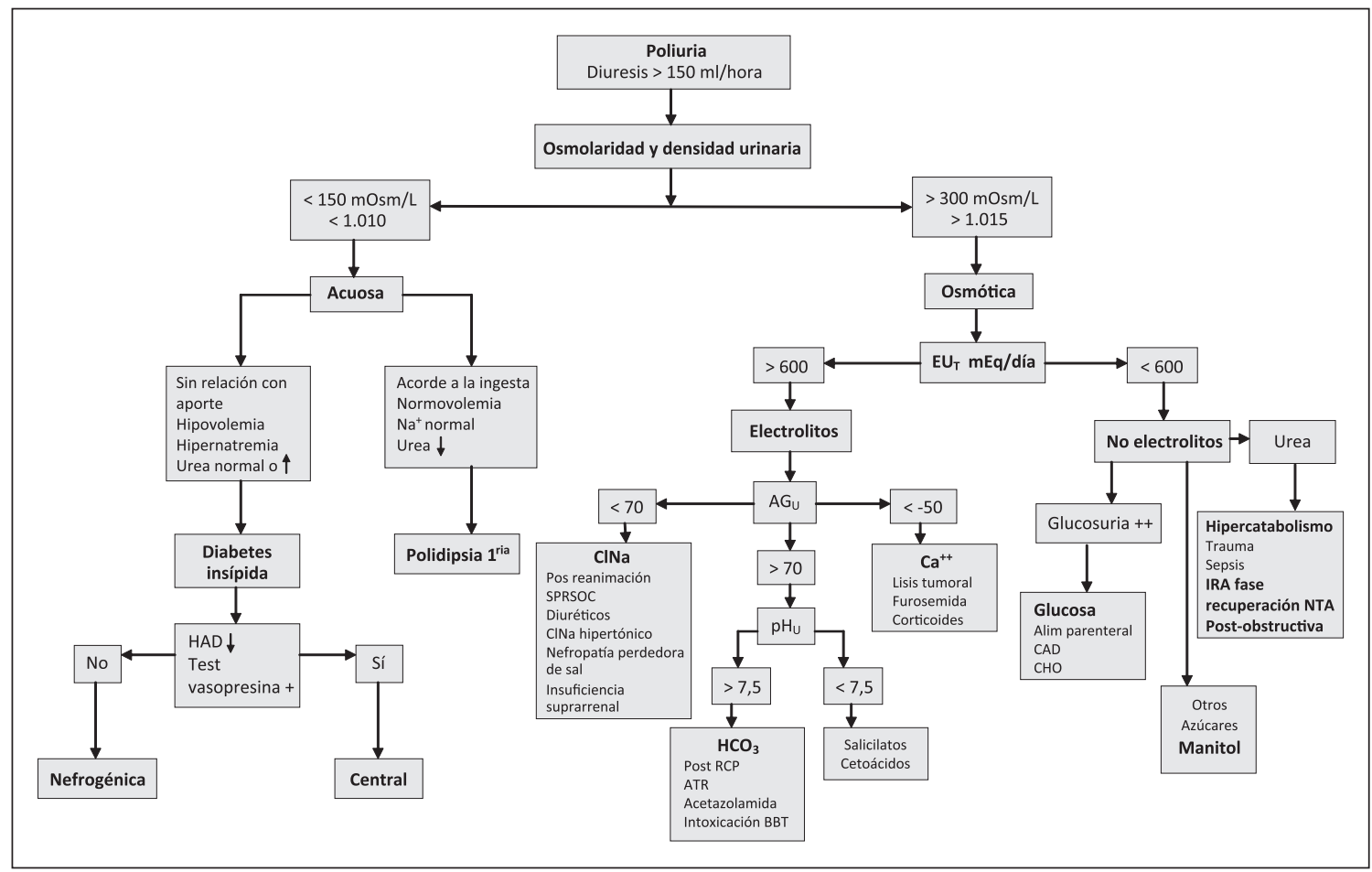

Figura 4. Algoritmo para el diagnóstico de los estados poliúricos. Abreviaturas: mosm/l: miliosmoles por litro; HAD: hormona antidiurética; EuT: electrolitos urinarios totales; AGu: Anion gap urinario; CINa. Cloruro de sodio; SPRSOC: síndrome de pérdida renal de sal de origen cerebral; $\mathrm{pHu}$ : $\mathrm{pH}$ urinario; $\mathrm{HCO}_{3}{ }^{-}$: bicarbonato; RCP: reanimación cardiopulmonar; ATR: acidosis tubular renal; BBT: barbitúricos; CAD. cetoacidosis diabética; CHO. coma hiperosmolar; IRA: injuria renal aguda; NTA: necrosis tubular aguda. 
- En aquellos casos en que poliuria se asocia a hiponatremia, descenso de la osmolaridad plasmática e hipovolemia, debe plantearse la posibilidad del síndrome de pérdida renal de sal de origen cerebral ${ }^{2,18,19,34,35}$. En estos casos están indicadas las soluciones salinas al 3\% y fludrocortisona con la finalidad de potenciar retención de agua y sodio ${ }^{2,18,19,34,35}$.

- El tratamiento de las poliurias hemodinámicas, se basa en descender presión de perfusión renal, a través de la disminución del aporte de fluidos ${ }^{2,8,10}$. Con estas medidas decrece la tasa de filtración glomerular y el flujo tubular de solutos ${ }^{2,8,10}$.

- Una práctica habitual que debiera abandonarse, es el aporte sistemático de desmopresina ante todo paciente con neuroinjuria grave y poliuria. La situación de mayor prevalencia es la poliuria osmótica con natremia normal o en descenso, por lo que el diagnóstico de DI central es muy poco probable, y el aporte de desmopresina no tiene fundamento alguno.

\section{Sexto paso: integración}

En la Figura 4, integramos los conceptos vertidos, de manera que el lector pueda disponer de una herramienta práctica a la hora de abordar el desafío diagnóstico de los estados poliúricos.

\section{Conclusiones}

Aunque desconocemos la incidencia de los estados poliúricos, sus consecuencias; hipovolemia, hipotensión, trastornos hidroelectrolíticos, son deletéreas y pueden conducir a daño encefálico secundario. Un enfoque ordenado y sistematizado poniendo énfasis en el cuadro clínico y los resultados del laboratorio permitirá determinar no sólo la etiología sino también la manera más eficaz de tratarlos.

\section{Referencias}

1. Cardoso APD, Dragosavac D, Araujo S, Falcao AL, Terzi RG, Castro M, et al. Syndromes related to sodium and arginine vasopressin alterations in post-operative neurosurgery. Arq Neuropsiquiatr 2007; 65 (3-B): 745-51.

2. Singh S, Bond D, Carlotti APCP, Cusimano M, Rutka J, Halperin ML. Cerebral Salt Wasting: Truths, fallacies, theories, and challenges. Crit Care Med 2002; 30: 2575 9 .

3. Blevins LS, Wand GS. Diabetes Insipidus. Crit Care Med 1992; 20: 69-79.

4. Edoute Y, Davids MR, Johnston C, Halperin ML. An integrative physiological approach to polyuria and hyponatremia: a 'double-take' on the diagnosis and therapy in a patient with schizophrenia. Q J Med 2003; 96: 531-40.

5. Robertson GL. Differential diagnosis of poliuria. Ann Rev Med 1988; 39: 425-42.

6. Oster JR, Singer I, Thatte L, Grant-Taylor I, Diego JM. The polyuria of solute diuresis. Arch Intern Med 1997; 157: 721-9.

7. Berl T, Schrier RW. Disorders of water metabolism. In: Schrier RW (Ed). Renal and electrolyte disorders. $5^{\text {th }} \mathrm{ed}$. Boston, Little, Brown, 1997: pp 1-87.

8. Kazda A, Jabor A, Zamecnik M, Masek K. Monitoring acid-base and electrolyte disturbances in Intensive Care. Adv ClinChem 1989; 27: 201-68.

9. Kumar S, Berl T. Disorders of water metabolism. In: Schrier RW, ed. Atlas of diseases of the kidney. Philadelphia: Blackwell, 1999: 1-22.

10. Halperin ML, Bohn D. Clinical approach to disorders of salt and water balance. Emphasis on integrative physiology. Crit Care Clin 18; 2002: 249-72.

11. Godoy DA, Videtta W, Piñero G. Injuria Cerebral Aguda. Abordaje Diagnóstico-Terapéutico Inicial. En Protocolos en Emergencias y Urgencias. Lizardi Pedro Editor. Manual Moderno. México, 2010, capítulo 3, pp. 15-20.

12. Anónimo. Poliuria-Wikipedia, la enciclopedia libre. 2012 http://es.wikipedia.org/wiki/Poliuria.

13. Chonchol M, Berl T, Melero R. Fisiología del agua y sodio. En: Agua, electrolitos y equilibrio ácido base. Ayus, Caramelo, Tejedor (Eds), Madrid, Editorial Médica Panamericana, 2006, pp 2-30.

14. Levin ER, Gardner DG, Samson WK. Natriuretic peptides. N Eng J Med 1998; 339: 321-8.

15. Harrigan MR. Cerebral salt wasting syndrome: a review. Neurosurgery1996; 38: 152-60.

16. Godoy DA, Manzi R, Piñero G. Enfermedad Cerebrovascular Isquémica, en Neurotrauma y neurointensivismo, Capítulo 48, pp. 587-607, Rubiano A, Pérez Yepes R, Eds. Distribuna, Bogotá. Colombia, 2007.

17. Rosner MJ. Pathophysiology and management of increased intracranial pressure. In Andrews BT. Neurosurgical Intensive Care, Mc Graw Hill Eds 1993; pp 57-112.

18. Rabinstein AA, Widjicks EF. Hyponatremia in critically ill neurological patients. Neurologist 2003; 9: 290-300.

19. Tisdall M, Crocker M, Watkiss J, Smith M. Disturbances 
of sodium in critically ill adult neurologic patients: A clinical review. J Neurosurg Anesthesiol 2006; 18: 57-63.

20. Bazerque F, Kairiyama O. Síndromes hiperosmolares. En Terapia intensiva, 3ra edición, sección VI, capítulo 8, pp. 638-47. Sociedad Argentina de Terapia Intensiva, Editorial Médica Panamericana, Buenos Aires 2000.

21. Rose BD, Post TW. Situaciones de hiperosmolaridad hipernatremia. En Rose and Post Eds. Trastorno de los electrolitos y del equilibrio ácido-base. Madrid, Editorial Marbán, 2002.

22. Ober KP. Diabetes Insipidus. Crit Care Clinics 1991; 7: 109-25.

23. Singer I, Oster J, Fishman LM. The Management of Diabetes Insipidus in adults. Arch Intern Med 1997; 157: 1293-901.

24. Cooperman M. Diabetes Insipidus: treatment \& management, June 2011. http://emedicine.medscape.com/ article/117648-treatment

25. Tsagarakis S, Tzanela M, Dimopoulou I. Diabetes insipidus, secondary hypoadrenalism and hypothyroidism after traumatic brain injury: clinical implications. Pituitary 2005; 8: 251-4.

26. Hannon MJ, Sherlock M, Thompson CJ. Pituitary dysfunction following traumatic brain injury or subarachnoid haemorrhage - in "Endocrine Management in the Intensive Care Unit”. Best Pract Res Clin Endocrinol Metab 2011; 25: 783-98.

27. Adrogué H, Madias NE. Hypernatremia. N Engl J Med 2000; 342: 1493-9.

28. Allen I, Ayus C. Pathogenesis and management of hypernatremia. Curr Opin Crit Care 1996; 2: 418-23.

29. Hans P, Bonhomme V, Damas F. Les hypernatrémies en pathologieneurochirurgicale. Ann Fr Anesth Réanim 2001; 20: 213-8.

30. Stelfox HT, Ahmed SB, Khandwala F, Zygun D, Shahpori R, Laupland K. The epidemiology of intensive care unit-acquired hyponatremia and hypernatremia in medical-surgical intensive care units. Crit Care 2008; R162.

31. Lindner G, Funk GC, Schwarz C, Kneidinger N, Kaider
A, Schneeweiss B, et al. Hypernatremia in the critically ill is an independent risk factor for mortality. Am J Kidney Dis 2007; 50: 952-7.

32. Ertmer C, Kampmeier T, Rehberg S, Lange M. Fluidresuscitation in multiple trauma patients. Curr Opin Anaesthesiol. 201; 24: 202-8.

33. Santry HP, Alam HB. Fluid resuscitation: past, present, and the future. Shock 2010; 33: 229-41.

34. Harrigan MR. Cerebral salt wasting syndrome: a review. Neurosurgery 1996; 38: 152-60.

35. Overgaard-Steensen C. Initial approach to the hyponatremic patient. Acta Anaesthesiol Scand 2011; 55: 139-48.

36. Upadhyay UM, Gormley WB. Etiology and management of hyponatremic neurosurgical patient. J Intensive Care Med 2011; Epub ahead of print. PMID 21345881.

37. Froelich M, Ni Q, Wess C, Ougorets I, Hartl R. Continuous hypertonic saline therapy and the occurrence of complications in neurocritically ill patients. Crit Care Med 2009; 37: 1433-41.

38. Paczynski RP. Osmotherapy: Basic concepts and controversies. Crit Care Clin 1997; 13: 105-29.

39. Castillo LB, Bugedo GA, Paranhos JL. Mannitol or hypertonic saline for intracranial hypertension?. A point of view. Crit Care Resuc 2009; 11: 151-4.

40. Brain Trauma Foundation; American Association of Neurological Surgeons; Congress of Neurological Surgeons; Joint Section on Neurotrauma and Critical Care, AANS/CNS. Guidelines for the management of severe traumatic brain injury. II. Hyperosmolar therapy. J Neurotrauma 2007; 24 Suppl 1: S14-20.

41. Electrolitos urinarios. Blog del químico clínico, junio de 2012. www.quimicoclinico.wordpress.com/2008/04/14/ electrolitos-urinarios/

42. Black RM. Disorders of plasma sodium and plasma potassium. In: Intensive care medicine. Irwin RS, Cerra FB, Rippe JM, Eds. $4^{\text {th }}$ ed. Philadelphia, Lippincott/Raven, 1999; 951-2.

43. Kumar S, Berl T. Sodium. The Lancet 1998; 352: 2208. 